\title{
Avaliação Parasitológica de Couve Minimamente Processada: Riscos Associados ao seu Consumo in Natura
}

\author{
Ariana Brito Santos ${ }^{l}$; Poliana Fernandes Gonçalves ${ }^{l}$; Josiane Sousa da Silva ${ }^{l}$; \\ Mariele Oliveira Aguiar ${ }^{l}$; Karine Silva Santos ${ }^{l}$; Rita Leite Queiroz ${ }^{1}$; Larissa Costa Silva Fogaça ${ }^{2}$
}

\begin{abstract}
Resumo: O número de pessoas que consomem hortaliças in natura contaminadas por parasitas, tem se elevado. Essa situação se dá principalmente, pela a falta de uma preparação e higienização da maneira correta. Neste contexto, objetivou-se avaliar a qualidade de couves (Brassica oleracea) minimamente processadas estabelecendo os riscos associados ao seu consumo in natura. As amostras foram coletadas em feiras livres da cidade de Vitória da Conquista - Bahia e avaliadas quanto à contaminação parasitológica pelo método de Hoffman, Pons e Janer (1934), popularmente conhecido coo sedimentação espontânea. As principais espécies encontradas nas amostras avaliadas foram Entamoeba coli, Ascaris lumbricoides; Giárdia lamblia; Taênia sp e Hymenolepis nana, corrobora, ainda, para a contaminação dos procedimentos indevidos de cultivo e qualidade insatisfatória de manipulação, evidenciando a necessidade de capacitações por meio de cursos de boas práticas de manipulação aos comerciantes com intuito de assegurar a qualidade dos produtos manipulados e a educação continuada a todos que trabalham nessa área, desde o cultivo até a comercialização.
\end{abstract}

Palavras-chave: Minimamente Processados. Enteroparasitas. Manipulação. Doenças Transmitidas por Alimentos.

\section{Parasitological Evaluation of Minimally Processed Cabbage: Risks Associated with its Consumption in Natura}

\begin{abstract}
The number of people consuming fresh vegetables contaminated by parasites has risen. This situation is mainly due to the lack of proper preparation and sanitation. In this context, the objective was to evaluate the quality of freshly bred cabbages (Brassica oleracea), establishing the risks associated with their in natura consumption. Samples were collected at free fairs in the city of Vitória da Conquista - Bahia and evaluated for parasitological contamination by the method of Hoffman, Pons and Janer (1934), popularly known as spontaneous sedimentation. The main species found in the samples evaluated were Entamoeba coli, Ascaris lumbricoides; Giárdia lamblia; Taenia sp and Hymenolepis nana, also corroborates the contamination of undue cultivation procedures and poor handling quality, evidencing the need for training by means of courses of good practices of manipulation to merchants in order to ensure the quality of the products handled and continuing education for all who work in this area, from cultivation to commercialization.
\end{abstract}

Keywords: Minimally processed. Enteroparasites. Manipulation. Foodborne Illness.

\footnotetext{
${ }^{1}$ Faculdade Independente do Nordeste. Contato: aribritosantos@gmail.com;

${ }^{2}$ Graduada em Engenharia de Alimentos e Mestre em Engenharia de Alimentos, pela Universidade Estadual do Sudoeste da Bahia. Professora na Faculdade Independente do Nordeste - Fainor, lotada no Colegiado de Farmácia.
} 


\section{Introdução}

Segundo a Organização Mundial de Saúde (OMS), estimase, anualmente, 1.8 milhões de mortes decorrentes de doenças diarréicas, em sua grande maioria, ligadas ao consumo de alimentos ou água contaminados. as parasitoses são as doenças mais comuns do mundo, atingem cerca de $50 \%$ da população mundial e são responsáveis por relevantes consequências na saúde de adultos e crianças (Program \& OMS, 2006). No Brasil, até 36\% da população tem alguma parasitose e, considerando só as crianças, a prevalência aumenta para 55,3\% (DUARTE, 2017).

As parasitoses intestinais são um problema de saúde pública, caracterizadas por uma alta prevalência e distinção das manifestações clínicas. A contaminação de hortaliças consumidas cruas é um das causas mais relevantes na epidemiologia das enteroparasitoses. A transmissão desses parasitas para o homem ocorre através do consumo de alimentos, especialmente vegetais in natura, que também incluem frutas e verduras (MOURA, SANTOS e SANTOS, 2015).

A infecção parasitária não depende exclusivamente do alimento, mas alguns procedimentos relacionados ao seu cultivo, tais como o solo e a água utilizados, o armazenamento, transporte, procedimentos ineficazes de higienização de manipuladores e utensílios utilizados no preparo desses vegetais (ANDRADE et al., 2013). Estes fatores isolados ou em conjunto tornam os alimentos minimamente processados consumidos no seu estado natural, importantes meios de transmissão.

As hortaliças minimamente processadas são aquelas preparadas por uma ou mais operações que incluem o descascamento e fatiamento, quando necessários. A cultura de alimentos já processados é realizada desde as duas últimas décadas no Brasil (GENZ, OLIVEIRA, DAGMAR, e RENNER, 2017; NASCIMENTO e ALENCAR, 2014). A mudança dos hábitos alimentares dos consumidores que optam por uma alimentação mais saudável, aliada a maior praticidade, tornam estes produtos mais atraentes ao consumo devido à facilidade que oferecem (PEIXOTO et al., 2014; PRADO et al., 2008).

Por ser um alimento barato de fácil acesso, rico em vitaminas, minerais, fibras, e seus valores nutricionais, oferece vários benefícios, a couve é uma hortaliça amplamente consumida, porém a falta de boas práticas de manipulação e cultivo podem torná-la importante 
veículo de várias enfermidades entéricas, como por exemplo: bactérias e vírus. Entretanto estruturas parasitárias se destacam os helmintos e protozoários, parasitas que acometem pessoas de todas as idades causando anemia, má absorção de nutrientes, diarreia, emagrecimento, diminuição na capacidade de aprendizado e trabalho, além diminuição no desenvolvimento físico e cognitivo, no caso das crianças (PEZZIN, 2017; E. P. SILVA, APARECIDA, COSTA, e SOARES, 2015).

Diante do exposto, o estudo objetiva-se avaliar a qualidade higiênico-sanitária de couves minimamente processadas, comercializadas em feiras livres da cidade de Vitória da Conquista Bahia, por meio de análises parasitológicas, visando à determinação de possíveis focos de contaminação durante o processamento dessas hortaliças, estabelecendo os riscos associados ao consumo destes.

\section{Metodologia}

A metodologia da investigação baseia-se na pesquisa empírica de amostras, recolhidas de modo aleatório, realizando-se a análise subsequente, com intuito de avaliar contaminação destas por parasitas.

Foram analisadas amostras de couves (Brassica oleracea.) frescas minimamente processadas, em feiras livres de Vitória da Conquista - Bahia, que foram obtidas no mês de agosto de 2018.

O procedimento de coleta das amostras foi realizado pelo recolhimento de uma amostra semanal, durante quatro semanas, em cada uma das cinco feiras livres, totalizando vinte amostras. É importante frisar que a coleta foi realizada de modo aleatório e às cegas, não havendo qualquer preparação ou alteração da oferta comum com que essas couves são oferecidas ao público consumidor. Por razões éticas, os comerciantes das couves minimamente processados não foram identificados, e as Feiras Livres onde foram coletadas as amostras foram decodificadas como os símbolos F1, F2, F3 e F4.

As amostras foram analisadas no laboratório de parasitologia da Faculdade Independente do Nordeste - FAINOR, conforme método baseado na técnica de sedimentação espontânea de Hoffman, Pons e Janer de 1934; Foram acondicionadas 200 
gramas de cada amostra em sacos plásticos estéreis juntamente com 250 mililitros de água destilada, em seguida, agitou-se vigorosamente por alguns minutos para que a água entrasse em contato com toda a amostra e para que removesse os possíveis agentes parasitários presentes. O liquido obtido foi filtrado em tamiz, recoberto com gaze dobrada em quatro partes, sob um cálice e deixado em repouso por 2 horas até completa sedimentação.

Ao fim do processo, uma gota do sedimento foi transferida para uma lâmina e acrescentada uma gota de lugol, sobrepondo uma lamínula. Prepararam-se lâminas em duplicata que foram observadas em microscópio óptico nas objetivas com aumento de 10x e 40x com o objetivo de avaliar a presença ou não de estruturas parasitárias. Os resultados foram obtidos e tabulados numa planilha do Excel, para uma melhor interpretação.

\section{Resultados e Discussão}

Dentre as amostras de couve analisadas, observou-se que $100 \%$ das amostras apresentaram contaminação parasitológica nas condições do experimento. As espécies mais frequentes de parasitos encontrados foram cistos de Entamoeba coli; Cistos de Giárdia lamblia; Ovos de Ascaris lumbricoides; Ovo de Taênia sp e ovos de Hemynolepis nana, conforme resultados apresentados na Tabela 1.

Tabela 1. Estruturas parasitárias encontradas em amostras de couves minimamente processadas comercializadas em Feiras Livres de Vitória da Conquista- BA.

\begin{tabular}{lcccc}
\hline \multicolumn{1}{c}{ PARASITAS } & \multicolumn{3}{c}{ FEIRAS LIVRES } \\
\cline { 2 - 5 } & F1 & F2 & F3 & F4 \\
\hline Entamoema coli & + & + & + & - \\
Hymenolepsis nana & + & - & - & - \\
Giardia lamblia & + & + & + & + \\
Ascaris lumbricoides & + & + & - & + \\
Taenia sp & - & - & + & - \\
Triscstronylus & - & - & + & - \\
Eieria Bukidnonensis & - & - & + & \\
\hline Fonte: Dados
\end{tabular}

Fonte: Dados da pesquisa. 
Os resultados evidenciam a prevalência das espécies Entamoeba coli e Ascaris lumbricoides em 100\% das amostras avaliadas. De acordo com estudos realizados essas são as espécies de parasitos mais encontrados nas avaliações realizadas em proporções de protozoários/helmintos diferentes. É possível inferir que estes parasitos são naturalmente mais resistentes à sobrevivência no ambiente, seja em solo, por serem geohelmintos ou em água (Barboza \& Carvalho, 2016; Dufloth et al., 2014), explicando a sua prevalência em amostras obtidas em diferentes pontos de comercialização.

A espécie Entamoeba coli, é um organismo não patogênico e encontra-se entre os protozoários comensais intestinais que não constituem um agravo à saúde, no entanto, atuam como um forte indicador de contaminação fecal nas amostras analisadas (Dong et al., 2017). Os resultados encontrados indicam que a população pode estar exposta à contaminação fecal através do consumo destes alimentos, o que torna indispensável a reflexão sobre as condições sanitárias, práticas de cultivo e manipulação destes, principalmente do ponto de vista higiênico (NDIAYE, DIOUF, DIOUF, FAYE, e NDIAYE, 2014).

Já o helminto Ascaris lumbricoides encontrado também em todas as amostras analisadas, apresentam grande risco associado ao consumo, pois podem desenvolver um processo patológico no ser humano, a partir da ingestão de água ou alimentos contaminados com ovos larvados. Os sintomas mais frequentes são: desconforto abdominal, cólicas intermitentes, dor epigástrica e má digestão; dentre outras sintomatologias (COSTA, TEODORO, BAHIA-DE-OLIVEIRA, NUNES, e BARATA, 2018; Sá et al., 2017).

A Giardia lamblia é frequentemente veiculada pela a água, encontrada em 50\% das amostras avaliadas, tem sua transmissão atrelada às más condições de higiene e por seus cistos contaminantes poderem resistir às condições do meio ambiente por meses. Os sintomas que se expressam clinicamente no infectado mantém direta relação com a quantidade de cistos que a pessoa ingeriu, com as condições imunológicas do hospedeiro e com a duração da doença. A manifestação clínica da giardíase mais comum é a diarreia fétida e explosiva (E. P. SILVA et al., 2015).

Nas analises, foram identificados o ovo de Taenia sp, este parasita é leve e pode ser transportados pelo vento a longas distâncias provocando a contaminação das folhas (PIRES, 2008). O ovo de Taenia solium quando ingerido pode ocasionar a cisticercose humana, que 
provoca quadros de convulsões e hipertensão intracraniana.Não é possível dizer se os ovos encontrados eram de Taenia solium ou Taenia saginata, pois os mesmos são difíceis de distinção mesmo com o uso do microscópio (PONDJA et al., 2015).

Observou-se também a presença de formas evolutivas de parasitas de animais que comumente não afetam o homem. Foram encontrados ovos de Triscstronylus e Eimeria bukidnonensis nas amostras de couves pesquisadas. Essa situação pode ser explicada pelo fato da hortaliça estar exposta a vetores e veículos que transmitem diversas estruturas patogênicas. Água contaminada com dejetos fecais de outros animais, moscas, baratas, são exemplos de disseminadores mecânicos de parasitas.

De acordo com os dados adquiridos na coleta, pode-se verificar um percentual elevado de protozoários que provém do contato com resquícios fecais, o que comprova um quadro bastante preocupante de saúde pública e de controle da qualidade dos alimentos disponíveis ao público consumidor ao passo que há uma falha na higienização das hortaliças estudadas ou o método de higienização utilizado não foi totalmente eficaz (GONÇALVES, DA SILVA, e STOBBE, 2013).

Conforme a Agência Nacional de Vigilância Sanitária através da Resolução da Comissão Nacional de Normas e Padrões para Alimentos (CNNPA), as hortaliças devem estar ausentes de sujidades, parasitas e larvas para serem ofertadas para consumo humano. Embora a comercialização de frutas e hortaliças em feiras seja importante para economia e para a sociedade, não existe uma legislação específica para regulamentar à atividade. Os princípios seguidos se baseiam na RDC MS n²16 e na RDC MS n 275 as quais não são específicas para estes estabelecimentos (RIBEIRO e RODRIGUES, 2017).

É de grande valia para saúde pública a efetivação de pesquisas que auxiliam na avaliação das condições higiênico sanitárias dos alimentos de forma geral, para que ocorra melhoria na qualidade destes, contribuindo para a constatação de processos de produção ineficientes (OLIVEIRA, MELO, SANTOS, e SOUZA, 2013).

A elevada contaminação parasitária das hortaliças revela condições comuns a muitas outras regiões do país, como relatos presentes em outros estudos similares. O risco de contaminação humana pelo consumo das hortaliças depende, entre outros fatores, da frequência com que os cistos de protozoários e ovos de helmintos aparecem nesses alimentos (Peixoto et al., 2014; M. V. Silva et al., 2017). De acordo com os autores do estudo a couve apresenta-se 
como um eficiente instrumento para avaliação da presença de parasitas e suas especificidades pelo método de Hoffman, metodologia utilizada também no presente estudo (DE ANDRADE BERNAL FAGIANI et al., 2017; MOURA et al., 2015).

\section{Considerações Finais}

Os resultados evidenciam negligência no cultivo, transportes, armazenamento e a manipulação da hortaliça o que torna uma fonte de contaminação para o consumidor final. Fatores como a falta de infraestrutura e de saneamento básico, inadequação higiênica no manuseio, e preparação dos alimentos ofertados ao público, possibilitam o surgimento e disseminação de doenças provocadas pelos agentes parasitários, sendo, portanto, um problema de saúde pública e prevenção de qualidade de vida.

A couve minimamente processada requer higienização eficiente, não apenas com a lavagem e assepsia dos mesmos, bem como dos materiais e sua preparação, e também o uso de luvas e com a profilaxia para a manipulação desta hortaliça. Os manipuladores devem ser orientados a adotar práticas de higiene corretas durante todo o procedimento, assim como há a necessidade de maior fiscalização por parte dos órgãos reguladores municipais quanto à adoção de boas práticas no manuseio de alimentos minimamente processados comercializados em feiras livres.

\section{Referências}

ANDRADE, A. S. A., CARVAlhO, C. D., BRITO, A. M. G., JERALDO, V. L. S., OLIVEIRA, C. C. C., e MELO, C. M. O cuidado como elo entre a saúde e as infecções parasitárias em creches. Ciência, Cuidado e Saúde, 2013, 12(2), 257-265. https://doi.org/10.4025/cienccuidsaude.v12i2.13838.

BARBOZA, M. DE O., e CARVALHO, A. S. Análise Parasitológica de contaminantes de origem fecal em banheiros Femininos de uma Instituição de Ensino Superior do interior da Bahia. Id on Line Multidisciplinary and Psycology Journal, 2016, 10, 237-248. 
COSTA, M. A., TEODORO, L., BAHIA-DE-OLIVEIRA, G., NUNES, A. P. e BARATA, R. Intestinal parasites in paper money circulating in the city of Diamantina (Minas Gerais, Brazil). Research and Reports in Tropical Medicine, 2018, Volume 9, 77-80. https://doi.org/10.2147/RRTM.S157896.

DE ANDRADE BERNAL FAGIANI, M., NATSUMI TOGAWA, K., REGINATO MARTINS, T., TOSHIKO TASHIMA, N., LELI DILLIO, F., HENRIQUE NAHAS CHAGAS, P., e APARECIDA DA SILVA, M. Avaliação Microbiológica E Parasitológica De Produtos Minimamente Processados no Município de Presidente Prudente-SP. COLLOQUIUM VITAE, 2017, 9(2), 17-21. https://doi.org/10.5747/cv.2017.v09.n2.v195.

DONG, H., LI, J., QI, M., WANG, R., YU, F., JIAN, F., ... ZHANG, L. Prevalence, molecular epidemiology, and zoonotic potential of Entamoeba spp. in nonhuman primates in China. Infection, Genetics and Evolution, 2017, 54, 216-220. https://doi.org/10.1016/j.meegid.2017.07.002.

DUARTE, F. M. Percepção de manipuladores de alimentos sobre risco sanitário, 2017. https://doi.org/10.1055/s-2004-823144.

DUFLOTH, D. B., SILVA, C. M., PESSOA NOBRE DE LACERDA, A. S. S., VIÉGAS DA SILVA, S. F., RESENDE TEIXEIRA, K. T., RUIZ MONTEIRO, T. M., ... AGUIAR, V. M. Pesquisa Sobre a Contaminação De Hortaliças Por Ovos E Larvas De Nematódeos E Cistos De Protozoários Como Método De Estudo. Revista de Patologia Tropical, 2014, 42(4), 443-454. https://doi.org/10.5216/rpt.v42i4.27923.

GENZ, T. B., OLIVEIRA, C. F. DE, DAGMAR, J., e RENNER, P. Eficácia antibacteriana de agentes de limpeza na desinfecção de superfícies de consultórios odontológicos. Revista Passo Fundo, 2017, 22(2), 162-166. https://doi.org/http://dx.doi.org/10.5335/rfo.v22i2.6781 *

GONÇAlVES, R. M., DA SILVA, S. R. P. e STOBBE, N. S. FREQUÊNCIA DE PARASITOS EM ALFACES (Lactuca sativa) CONSUMIDAS EM RESTAURANTES selfservice DE PORTO ALEGRE, RIO GRANDE DO SUL, BRASIL. Revista de Patologia Tropical, 2013, 42(3), 323-330. https://doi.org/10.5216/rpt.v42i3.26926.

MOURA, L. R., SANTOS, T. e SANTOS, T. PESQUISA DE PARASITOS EM ALFACE E COUVE PROVENIENTES DE FEIRAS DA REGIÃO CENTRAL E SUAS MEDIAÇÕES NA CIDADE DE ANÁPOLIS-GO RESEARCH OF PARASITES IN LETTUCE AND CABBAGE FROM FAIRS IN THE CENTRAL REGION AND ITS MEDIATIONS IN THE CITY OF. Revista Educação Em Saúde, 2015, 3, 35-41.

NASCIMENTO, E. D. DO e ALENCAR, F. L. S. Eficiência Antimicrobiana E Antiparasitária De Desinfetantes Na Higienização De Hortaliças Na Cidade De Natal - Rn. Ciência e Natura, 2014, 36(2), 92-106. https://doi.org/10.5902/2179460X12755 
NDIAYE, A., DIOUF, M. N., DIOUF, M., FAYE, N., e NDIAYE, I. P. Epidemiological Profile of gastrointestinal parasites at Analysis Center of hospital Abass NDAO from 2011 to 2012. International Journal Current Microbiology And Apllied Sciences, 2014, 3(2), 1-8.

OLIVEIRA, M. DE C. M., MElO, D. S., SANTOS, J. A. C. DOS, e SOUZA, L. T. de. AVALIAÇÃO QUALITATIVA DAS PREPARAÇÕES DO CARDÁPIO DE UMA UNIDADE DE ALIMENTAÇÃO E NUTRIÇÃO INDUSTRIAL DE VITÓRIA DA CONQUISTA - BA, 2013, 38-42.

PEIXOTO, L. O., AZEVEDO, C. V. DE, ALMEIDA, S. M. DE A., FREITAS, B. K. S. DE, MELO, M. V. C., e SILVA, I. N. G. da. Avaliação microbiológica e parasitológica de alfaces minimamente processadas, comercializadas em supermercados da cidade de Fortaleza, Ceará. Nutrivisa: Revista de Nutrição e VigilâNcia Em Saúde, 2014, 1(1), 27-31. Retrieved from http://www.revistanutrivisa.com.br/artigo-original/avaliacao-microbiologica-e-parasitologicade-alfaces-minimamente-processadas-comercializadas-em-supermercados-da-cidade-defortaleza-ceara/

PEZZIN, J. Enteroparasites in vegetables marketed in an ancient Brazilian city. Rev. Salud Pública., 2017, 19(5), 635-640. https://doi.org/https://doi.org/10.15446/rsap.v19n5.57141

PONDJA, A., NEVES, L., MLANGWA, J., AFONSO, S., FAFETINE, J., WILLINGHAM, A. L., ... JOHANSEN, M. V. Incidence of porcine cysticercosis in Angónia District, Mozambique. Preventive Veterinary Medicine, 2015, 118(4), 493-497. https://doi.org/10.1016/j.prevetmed.2015.01.001.

PRADO, S. DE P. T., RIBEIRO, E. G. A., CAPUANO, D. M., AQUINO, A. L. DE, ROCHA, G. DE M., e BERGAMINI, A. M. M. Avaliação microbiológica, parasitológica e da rotulagem de hortaliças minimamente processadas comercializadas no município de Ribeirão Preto, SP/Brasil. Rev. Inst. Adolfo Lutz, 2008, 67(3), 221-227. Retrieved from http://internet.

PROGRAM, P. H., e OMS. Cinco chaves para uma alimentação mais segura manual. Oms, 2006, 1-29. https://doi.org/ISBN 9241594632.

RIBEIRO, D. F., \& RODRIGUES, R. da S. Avaliação das condições higiênico-sanitárias na comercialização de frutas e hortaliças no município de Manhuaçu, Minas Gerais Evaluation of the hygienic-sanitary conditions in the commercialization of fruits and vegetables in the city of Manhuaçu , Minas. Revista Verde de Agroecologia e Desenvolvimento Sustentável, 2017, 12(1), 85-89.

SÁ, M. F. DE, GONÇALVES, R. A., MARDER, C., BALDISSERA, M. D., OLIVEIRA, C. B. DE, NOLL, J. C. G., ... MONTEIRO, S. G. Adapted Bailenger method improves the rate of Ascaris suum eggs recovery from liquid pig manure compost. Ciência Rural, 2017, 47(4), 1-6. https://doi.org/10.1590/0103-8478cr20160837

SILVA, E. P., APARECIDA, R., COSTA, M., e SOARES, M. A. (2015). ASPECTOS HIGIÊNICO-SANITÁRIOS DE FEIRANTES E ANÁLISE PARASITOLÓGICA DE 
HORTIFRÚTISCOMERCIALIZADOS EM FEIRAS LIVRES DE MUNICÍPIOS DO ESTADO DE MINAS GERAIS, BRASIL. Revista Da Universidade Vale Do Rio Verde, 11(2), 591-602.

SILVA, M. V., PINTO, L. C., ARRAIS, F. M. DE A., COSTA, A. R. DE S., SILVA, M. J. R. DA, e FERREIRA, R. J. (2017). Estudo parasitológico de alface (Lactuca sativa L.) em alimentos fast food comercializados em festas populares do Cariri. Biota Amazônia, 7(3), 2832. Retrieved from https://periodicos.unifap.br/index.php/biota/article/view/2738/v7n3p2832.pdf.

\section{Como citar este artigo (Formato ABNT):}

SANTOS, Ariana Brito; GONÇALVES, Poliana Fernandes; SILVA, Josiane Sousa da; AGUIAR, Mariele Oliveira; SANTOS, Karine Silva; QUEIROZ, Rita Leite; FOGAÇA, Larissa Costa Silva. Avaliação Parasitológica de Couve Minimamente Processada: Riscos Associados ao seu Consumo in Natura. Id on Line Rev.Mult. Psic., 2018, vol.12, n.42, p. 933-942, 2018. ISSN: 1981-1179.

Recebido: 26/10/2018;

Aceito: $27 / 10 / 2018$ 